\title{
Patient and parental assessment of factors influencing the choice of treatment in pediatric hydrocephalus
}

\author{
Mandeep S. Tamber, MD, PhD, ${ }^{1}$ and Robert P. Naftel, MD² \\ ${ }^{1}$ British Columbia Children's Hospital, University of British Columbia, Vancouver, British Columbia, Canada; and ${ }^{2}$ Vanderbilt \\ University Medical Center, Nashville, Tennessee
}

\begin{abstract}
OBJECTIVE Choosing between competing options (shunt or endoscopic third ventriculostomy) for the management of hydrocephalus requires patients and caregivers to make a subjective judgment about the relative importance of risks and benefits associated with each treatment. In the context of this particular decision, little is known about what treatmentrelated factors are important and how they are prioritized in order to arrive at a treatment preference.

METHODS The Hydrocephalus Association electronically distributed a survey to surgically treated hydrocephalus patients or their families. Respondents rated the importance of various surgical attributes in their decision-making about treatment choice, and also indicated their preference in hypothetical scenarios involving a trade-off between potential risks and benefits of treatment. Rank-order correlations were used to determine whether certain predictor variables affected the rating of factors or hypothetical treatment choice.
\end{abstract}

RESULTS Eighty percent of 414 respondents rated procedural risks, minimizing repeat surgery, and improving longterm brain function as being very or extremely important factors when deciding on a treatment; $69 \%$ rated the need to implant a permanent device similarly. Parent-respondents rated procedural risks higher than patient-respondents. A majority of respondents $(n=209,54 \%)$ chose a procedure with higher surgical risk if it meant that implantation of a permanent device was not required, and respondents were more likely to choose this option if they discussed both treatment options with their surgeon prior to their initial intervention (Spearman rho 0.198, $p=0.001$ ).

Although only 144 of 384 total respondents (38\%) chose a less established operation if it meant less repeat surgery, patient-respondents were more likely to choose this option compared to parent-respondents (Spearman rho 0.145, $p=$ 0.005). Likewise, patient-respondents were more likely than parent-respondents to choose an operation that involved less repeat surgery and led to worse long-term brain function (Spearman rho 0.160, $p=0.002$ ), an option that was chosen by only $23(6 \%)$ of respondents overall.

CONCLUSIONS This study is the first exploration of patient/parental factors that influence treatment preference in pediatric hydrocephalus. Procedural risks, minimizing repeat operations, and the desire to maximize long-term cognitive function appeared to be the most important attributes that influenced treatment decisions that the survey respondents had made in the past. Patients and/or their caregivers appear to see some inherent benefit in being shunt free. It appears that fear of multiple revision operations may drive treatment choice in some circumstances.

https://thejns.org/doi/abs/10.3171/2020.5.PEDS2095

KEYWORDS cerebrospinal fluid shunt; hydrocephalus; medical decision-making; pediatric; treatment; ventriculostomy

$\mathrm{C}$ EREBROSPINAL fluid shunt placement and endoscopic third ventriculostomy (ETV), with or without choroid plexus cauterization (CPC), represent mainstay surgical treatments for pediatric hydrocephalus. Each surgical procedure has its own profile with respect to the potential for harm (risk) and clinical effectiveness (benefit). Although concrete data are available for traditional surgical outcomes such as revision rates and infection, much uncertainty remains regarding the frequency of other surgical risks and, perhaps most importantly, around the question of which surgical procedure is superior with respect to long-term neurodevelopmental outcome and quality of life.

Procedural failure, necessitating repeat surgery, occurs in approximately $40 \%$ of patients within the first year following initial CSF shunt placement or ETV ${ }^{1,2}$ The temporal character of initial treatment failure, however, appears to differ between these two options for CSF diversion. ETV 
failures rapidly accrue in the first 60 days following the initial procedure, but thereafter the probability of failure is lower. This is in contrast to patients with a CSF shunt who continue to accumulate failures at a fairly uniform rate over the first 2 years following initial device placement. Taken together, this information may suggest that a long-term treatment advantage for ETV over CSF shunting exists after the early high-risk period of ETV failure, with 5-year failure rates for ETV and CSF shunting being estimated at $48 \%$ and $60 \%$, respectively. ${ }^{3,4}$ Initial experience with ETV-CPC in a North American setting appears to suggest a higher failure rate than CSF shunting in the short term (6 months) ${ }^{5,6}$ its long-term performance compared to shunt procedures and ETV remains uncertain.

The best-characterized surgical risk related to CSF diversionary procedures is infection, with rates ranging from $3 \%$ to $12 \%$ for CSF shunt procedures ${ }^{7}$ and from $2 \%$ to $4 \%$ for ETV (with or without CPC)., ${ }^{2,6}$ Unfortunately, robust comparative data concerning the frequency of other procedural risks with CSF shunting and ETV (such as hemorrhage, brain injury, and seizures) are lacking. Although the infection risk is lower with ETV, most contemporary data point toward a slightly higher procedure-related risk associated with endoscopic procedures compared to shunt operations. ${ }^{8,9}$

The question of whether neurodevelopmental outcome and/or quality of life is affected by the choice of initial treatment for pediatric hydrocephalus also remains largely unanswered. In the setting of treatment of postinfectious hydrocephalus in infants in sub-Saharan Africa, no significant difference in cognitive outcome was observed between CSF shunt placement and ETV-CPC..$^{10}$ A recent evaluation of 5-year health outcomes following initial treatment of infant hydrocephalus secondary to aqueductal stenosis demonstrated no significant difference in health status and quality of life between those infants treated with ETV and CSF shunt placement. ${ }^{11}$ Equivalent health outcomes between ETV and CSF shunting were also observed in a decision analysis. ${ }^{3}$

Given that there is no clear profile of procedural risk and benefit that would lead to a declaration that one form of CSF diversion is superior to another, uncertainty regarding the best surgical option for the initial treatment of pediatric hydrocephalus remains. As a result, treatment choice is influenced by the preferences of the patient, their family, and their surgeon. Choosing between two competing treatments requires patients and/or their caregivers to make a subjective assessment about the relative importance of the risks and benefits associated with each alternative treatment modality. The willingness of decisionmakers to accept treatment-related risks in exchange for treatment benefits represents a trade-off that can be quantified using preference methods based on welfare theory. ${ }^{12}$ Prior to the application of standard gamble or threshold methodologies, it is necessary to elicit which attributes of a particular treatment will factor into a person's decisionmaking framework. In the context of surgery for pediatric hydrocephalus, little is known about what treatment-related factors are important and how they are prioritized in order to arrive at a treatment preference. The purpose of this study was to enumerate the issues that patients and their families confront when deciding between CSF shunt placement and endoscopic surgery for the initial treatment of childhood hydrocephalus.

\section{Methods \\ Study Sample and Survey Methodology}

Information from a convenience sample of patients or family members of patients who underwent the first treatment of their hydrocephalus prior to the age of 18 years was gathered using an electronic survey distributed by the Hydrocephalus Association. The survey was posted to the Hydrocephalus Association Facebook page between September 23, 2016, and October 8, 2016. At the time, the Hydrocephalus Association had 27,600 Facebook followers. An introductory paragraph specifying the target group for this survey (individuals or parents of individuals who received their first treatment of hydrocephalus prior to the age of 18 years) was followed by a link to the survey itself. Of the Facebook group's followers, 1270 (4.6\%) individuals clicked on the link to view the survey. Because participation in this survey was voluntary and anonymous without the need to query health records, no IRB approval was required.

Demographic variables collected included the category of participant (patient or parent); the current treatment for hydrocephalus (shunt or endoscopy); the initial treatment for hydrocephalus (shunt or endoscopy); the age at initial treatment ( $<1$ year, $1-2$ years, $3-10$ years, or 11-18 years); the number of repeat (revision) operations $(0,1-5,6-10$, or $>10$ ); and whether their treating surgeon discussed both CSF shunt placement and endoscopy (ETV [with or without CPC]) prior to deciding on initial treatment (yes or no).

Participants were then asked to rate the importance of the following attributes related to surgery for CSF diversion by using a 5-point Likert scale (i.e., not at all important, slightly important, moderately important, very important, or extremely important): procedural risks (risk of wound [skin] infection, risk of brain infection [meningitis], risk of brain bleeding, risk of brain injury, risk of seizure, or risk of stroke); need to implant a permanent device; knowing immediately if the treatment is working; minimizing the need for repeat surgery over the next few months; minimizing the need for repeat surgery throughout a lifetime; improving long-term brain function; undergoing an older (more established) surgery; and undergoing a newer procedure with less known about it. In order to ensure that relevant attributes were not excluded by the investigators, this section of the survey ended with a freetext box asking participants if any other factors influenced their decision-making in this context.

\section{Modified Discrete Choice Experiment}

The concluding section of the survey asked participants whether they would accept a hypothetical treatment that had a perceived risk attribute coupled with a perceived benefit attribute. Participants were asked whether they would accept an operation with the following attributes: with higher surgical risk if it led to better long-term brain function; with higher surgical risk if it avoided the need to implant a permanent device; with unknown risks/ben- 
TABLE 1. Rating of various attributes related to surgery for CSF diversion

\begin{tabular}{|c|c|c|c|c|c|}
\hline Attribute & $\begin{array}{c}\text { Not } \\
\text { Important }\end{array}$ & $\begin{array}{l}\text { Slightly } \\
\text { Important }\end{array}$ & $\begin{array}{c}\text { Moderately } \\
\text { Important }\end{array}$ & $\begin{array}{c}\text { Very } \\
\text { Important }\end{array}$ & $\begin{array}{l}\text { Extremely } \\
\text { Important }\end{array}$ \\
\hline Risk of wound (skin) infection & $16(4 \%)$ & $48(12 \%)$ & $87(21 \%)$ & $113(27 \%)$ & $150(36 \%)$ \\
\hline Risk of brain infection (meningitis) & $9(2 \%)$ & $10(2 \%)$ & $17(4 \%)$ & $76(18 \%)$ & $302(73 \%)$ \\
\hline Risk of brain bleeding & $5(1 \%)$ & $8(2 \%)$ & $15(4 \%)$ & $63(15 \%)$ & $323(78 \%)$ \\
\hline Risk of brain injury & $3(1 \%)$ & $6(1 \%)$ & $15(4 \%)$ & $46(11 \%)$ & $344(83 \%)$ \\
\hline Risk of seizure & $8(2 \%)$ & $18(4 \%)$ & $41(10 \%)$ & $94(23 \%)$ & $253(61 \%)$ \\
\hline Risk of stroke & $6(1 \%)$ & $16(4 \%)$ & $18(4 \%)$ & $62(15 \%)$ & $312(76 \%)$ \\
\hline Need to implant a permanent device & $23(6 \%)$ & $36(9 \%)$ & $69(17 \%)$ & $97(23 \%)$ & $189(46 \%)$ \\
\hline Need to know immediately if the treatment is working & $3(1 \%)$ & $14(3 \%)$ & $43(10 \%)$ & $115(28 \%)$ & $239(58 \%)$ \\
\hline $\begin{array}{l}\text { Minimizing the need for repeat surgery over the next } \\
\text { few mos }\end{array}$ & $3(1 \%)$ & $2(0 \%)$ & $27(7 \%)$ & $75(18 \%)$ & $307(74 \%)$ \\
\hline Minimizing the need for repeat surgery over a lifetime & $4(1 \%)$ & $3(1 \%)$ & $30(7 \%)$ & $78(19 \%)$ & $299(72 \%)$ \\
\hline Improving long-term brain function & $3(1 \%)$ & $4(1 \%)$ & $8(2 \%)$ & $61(15 \%)$ & $338(82 \%)$ \\
\hline Undergoing an older, more established surgery & $17(4 \%)$ & $48(12 \%)$ & $137(33 \%)$ & $96(23 \%)$ & $116(28 \%)$ \\
\hline Undergoing a newer procedure with less known about it & $31(8 \%)$ & $56(14 \%)$ & $140(34 \%)$ & $80(19 \%)$ & $107(26 \%)$ \\
\hline
\end{tabular}

Values are presented as number (\%). Percentages may not sum to $100 \%$ due to rounding.

efits if it avoided implanting a permanent device; that may require more repeat surgeries if it led to better long-term brain function; that may require fewer repeat operations but lead to worse long-term brain function; with unknown risks/benefits if it may require less repeat surgery; with higher surgical risk if it led to worse long-term brain function; or with lower surgical risk if it led to better long-term brain function. The last two questions were included as a screen to ensure that participants were interpreting and answering these questions appropriately.

\section{Statistical Analysis}

Data are reported as frequencies and percentages where appropriate. Likert scale responses were treated as an ordinal variable for analysis. The ordinal-by-ordinal associations between demographic variables and the participants' rating of attributes related to surgery for CSF diversion, and the associations between demographic variables and whether or not the participant would choose a particular hypothetical treatment for childhood hydrocephalus were explored using Spearman's rank-order correlation coefficient. All analyses were conducted using the Statistical Package for the Social Sciences (SPSS) version 25 (IBM Corp.). We chose for significance a $\mathrm{p}$ value < 0.005 , a value that was believed to balance the number of comparisons performed with the exploratory nature of this work.

\section{Results}

\section{Characteristics of Respondents}

There were 438 total responses; 427 participants answered all of the demographic questions. The majority of respondents were parents of children with hydrocephalus ( $\mathrm{n}=292,70 \%)$. The most common clinical scenario was that of an infant less than 1 year of age treated initially with a CSF shunt. Approximately $25 \%(\mathrm{n}=99)$ of respon- dents had no prior revision surgery, $50 \%(\mathrm{n}=214)$ had between 1 and 5 revision operations, and the remaining $25 \%$ $(n=125)$ had 6 or more revisions following their initial CSF diversionary procedure. Of the 357 respondents who could recall whether or not they discussed the advantages and disadvantages of both CSF shunt and endoscopic surgery with their surgeon prior to making their initial treatment decision, only $32 \%$ answered in the affirmative.

\section{Rating the Importance of Attributes in Decision-Making}

There were 414 respondents who rated various attributes related to surgery for CSF diversion by using a 5-point Likert scale (Table 1). Most of the procedural risks, with the exception of wound infection, were rated very important or extremely important by more than $80 \%$ of participants. Other attributes that at least $80 \%$ of respondents judged to be very or extremely important in their decision-making included knowing immediately if the treatment is working, minimizing the need for repeat surgery in the short term as well as throughout a lifetime, and improving long-term brain function. The need to implant a permanent device was rated a very or extremely important factor in decision-making for $69 \%$ of respondents. When participants were asked whether there were any other factors that influenced their past decisions, no new attributes were identified.

The associations between demographic variables and the participants' rating of attributes related to surgery for CSF diversion revealed that all procedural risks were rated more highly by parent-respondents than by patientrespondents ( $\mathrm{p}<0.001$ for all procedural risks). Parentrespondents also rated minimizing the need for revision surgery over both the short term (Spearman rho 0.150, $\mathrm{p}=$ 0.003 ) and the long term (Spearman rho 0.188, p < 0.001), as well as improving long-term brain function (Spearman rho $0.193, \mathrm{p}<0.001$ ), more highly than did patient-respondents. The need to implant a permanent device demon- 
strated a trend toward significance (Spearman rho 0.138, $\mathrm{p}=0.006$ ), and once again it was rated more highly by parents than patient-respondents.

All procedural risks (with the exception of skin infection) were perceived to have less importance in decisionmaking as the number of revision operations increased ( $\mathrm{p}$ $<0.001$ for all procedural risks except skin infection). Alternatively, those who had not yet experienced a revision tended to rate procedural risks higher than those who had experienced at least one treatment failure in their lifetime.

Patient age at the time of first intervention, the initial or current CSF diversionary procedure, and whether or not the pros and cons of CSF shunt placement and ETV were discussed with the patient and/or their family before the first surgical intervention were not associated with the rating of attributes.

\section{Choices Made in Hypothetical Risk/Benefit Surgical Scenarios}

Overall, 384 (88\%) of respondents were able to respond to the series of questions asking whether they would choose a hypothetical surgical treatment for childhood hydrocephalus that had a specific procedural risk coupled with a specific procedural benefit. For the most part, the participants made choices that would maximize their utility, as evidenced by the fact that only $10(2.6 \%)$ respondents answered that they would not choose an operation with lower surgical risk if it led to better long-term brain function, and by the fact that only $24(6.25 \%)$ participants would choose an operation with higher surgical risk if it led to worse long-term brain function.

Operations that led to better long-term brain function seemed to be favored by respondents, even though they may have had higher procedural risk $(\mathrm{n}=292,76 \%)$ or require more revision operations $(n=267,69.5 \%)$. A majority of respondents $(n=209,54.4 \%)$ also chose a procedure with higher surgical risk if it meant that implantation of a permanent device was not required, and respondents were more likely to choose this option if they discussed both CSF shunt placement and ETV with their surgeon prior to the initial intervention (Spearman rho 0.198, $\mathrm{p}=0.001$ ).

The most notable association between demographic variables and the choice made between two hypothetical treatments for childhood hydrocephalus was found for operations that may lead to less repeat (revision) surgery. Although only 144 of 384 total respondents (38\%) chose a less established operation if it meant less repeat surgery, patient-respondents were more likely to choose this option compared to parent-respondents (Spearman rho 0.145, p $=0.005)$. Likewise, patient-respondents were more likely than parent-respondents to choose an operation that involved less repeat surgery and led to worse long-term brain function (Spearman rho 0.160, p = 0.002), an option that was chosen only by $23(6 \%)$ of respondents overall.

\section{Discussion}

This study represents the first exploration of patient and parental factors that influence treatment preference in the management of pediatric hydrocephalus. It provides unique insights into how procedural attributes may inter- act (in tangible and intangible ways) to affect the medical decision-making process from the patient and/or caregiver perspective.

Procedural risks, minimizing the need for repeat (revision) operations, and the desire to maximize long-term brain (i.e., cognitive) function appeared to be the most important attributes that influenced the treatment decisions that our survey respondents had made in the past-they were cited as being very important or extremely important by at least 4 of 5 participants.

Although participants did not rate the need to implant a permanent device as highly as some of the other treatment attributes, our results are still consistent with the notion that patients and/or their caregivers appear to see some inherent benefit in being shunt free. The respondents were asked to value this attribute without any specific reference being made to possibly increased risk of more repeat surgeries (e.g., for infection or mechanical complications). In this way, respondents were free to value this attribute according to whatever they felt was important to them, be it what they perceived to be an implant's relationship with the need for more surgery or other psychological effects associated with having an implanted device (e.g., having a "foreign" body within them, or having to rely on a "nonphysiologic" treatment for their condition). More than half of participants would choose an operation with higher procedural risk if it meant not having an implanted device. This finding is congruent with anecdotal observations that some families prefer an attempt at ETV-CPC (which has a higher procedural risk) over initial CSF shunt placement, despite evidence that suggests that treatment failure, at least in the short term, is higher for ETV-CPC.

It also appears that patients "fear" having multiple revision operations to the point of choosing a less established operation (e.g., ETV-CPC) or accepting a worse functional outcome. The fact that those respondents who have experienced many revisions tended to rate the importance of procedural risks lower than those respondents who have not experienced any revisions suggests that this fear is not related to procedural risks per se, but to other intangible aspects of having to undergo multiple repeat surgical interventions.

\section{Limitations of the Study}

This was a convenience sample drawn from a population that previously had undergone treatment for childhood hydrocephalus. As such, the responses from this group may not be representative of those from individuals who are contemplating this particular treatment decision prior to embarking on a surgical intervention for the first time.

The fact that survey participants were asked to think back to their initial treatment decision allows for the potential for cognitive dissonance to impact the responses that were provided. After a decision has already been made, cognitive dissonance may make participants rationalize the decision that they had made in the past, either by downplaying the positive attributes of the competing alternative or by exaggerating the negative attributes of the comparator.

In an attempt to explore the inherent biases of respon- 
dents as they worked through these hypothetical treatment choice scenarios ("signal"), we took steps to minimize any bias imparted by the investigators ("noise") on the responses of the participants. We tried to explore attitudes related to different procedures for hydrocephalus, including ETV-CPC, without biasing the respondent either in how the question was framed or by providing data suggesting that one procedure was superior to another in some attribute for which no such data exist. To the best of our ability in this particular forum, each respondent was allowed to interpret the question according to their own personal framework with minimal (albeit not zero) influence from outside sources.

These limitations speak to the need to undertake this type of study in a prospective fashion in a group of individuals who have yet to make a treatment decision.

\section{Conclusions}

This study represents the first exploration of patient/ parental factors that influence treatment preference in the management of pediatric hydrocephalus. The information presented here has many important applications. It will assist in the design of future discrete choice experiments, which will be used to define the threshold levels of minimum benefit and maximum risk(s) associated with shunt or ETV (with or without CPC) that are acceptable to patients. Evidence related to treatment preferences is also fundamental to the construction of decision aids relevant not only to the management of pediatric hydrocephalus but also to other pediatric neurosurgical conditions. Finally, patient preferences are invaluable to the design of highquality clinical studies. Some of the preferences expressed by our survey participants, specifically as they relate to the importance of maximizing long-term cognitive outcome following treatment for childhood hydrocephalus, led to the designation of neurocognitive development as the primary outcome of a Hydrocephalus Clinical Research Network randomized controlled trial comparing CSF shunt placement to ETV-CPC for the initial treatment of infant hydrocephalus.

\section{Acknowledgments}

We acknowledge the engagement of the survey participants who thoughtfully contributed their responses. We also acknowledge Jenna Koschnitzky, Jennifer Bechard, and Amanda Garzon at the Hydrocephalus Association for their invaluable assistance with this project.

\section{References}

1. Kulkarni AV, Riva-Cambrin J, Butler J, et al. Outcomes of CSF shunting in children: comparison of Hydrocephalus Clinical Research Network cohort with historical controls: clinical article. J Neurosurg Pediatr. 2013;12(4):334-338.

2. Kulkarni AV, Riva-Cambrin J, Holubkov R, et al. Endoscopic third ventriculostomy in children: prospective, multicenter results from the Hydrocephalus Clinical Research Network. J Neurosurg Pediatr. 2016;18(4):423-429.

3. Drake JM, Kulkarni AV, Kestle J. Endoscopic third ventriculostomy versus ventriculoperitoneal shunt in pediatric patients: a decision analysis. Childs Nerv Syst. 2009;25(4): 467-472.
4. Kulkarni AV, Drake JM, Kestle JR, et al. Endoscopic third ventriculostomy vs cerebrospinal fluid shunt in the treatment of hydrocephalus in children: a propensity score-adjusted analysis. Neurosurgery. 2010;67(3):588-593.

5. Kulkarni AV, Riva-Cambrin J, Browd SR, et al. Endoscopic third ventriculostomy and choroid plexus cauterization in infants with hydrocephalus: a retrospective Hydrocephalus Clinical Research Network study. J Neurosurg Pediatr. 2014; 14(3):224-229.

6. Kulkarni AV, Riva-Cambrin J, Rozzelle CJ, et al. Endoscopic third ventriculostomy and choroid plexus cauterization in infant hydrocephalus: a prospective study by the Hydrocephalus Clinical Research Network. J Neurosurg Pediatr. 2018; 21(3):214-223.

7. Kestle JR, Riva-Cambrin J, Wellons JC III, et al. A standardized protocol to reduce cerebrospinal fluid shunt infection: the Hydrocephalus Clinical Research Network Quality Improvement Initiative. J Neurosurg Pediatr. 2011;8(1):22-29.

8. Bouras T, Sgouros S. Complications of endoscopic third ventriculostomy. J Neurosurg Pediatr. 2011;7(6):643-649.

9. Wong JM, Ziewacz JE, Ho AL, et al. Patterns in neurosurgical adverse events: cerebrospinal fluid shunt surgery. Neurosurg Focus. 2012;33(5):E13.

10. Kulkarni AV, Schiff SJ, Mbabazi-Kabachelor E, et al. Endoscopic treatment versus shunting for infant hydrocephalus in Uganda. N Engl J Med. 2017;377(25):2456-2464.

11. Kulkarni AV, Sgouros S, Leitner Y, Constantini S. International Infant Hydrocephalus Study (IIHS): 5-year health outcome results of a prospective, multicenter comparison of endoscopic third ventriculostomy (ETV) and shunt for infant hydrocephalus. Childs Nerv Syst. 2018;34(12):2391-2397.

12. Hauber AB, Fairchild AO, Johnson FR. Quantifying benefitrisk preferences for medical interventions: an overview of a growing empirical literature. Appl Health Econ Health Policy. 2013;11(4):319-329.

\section{Disclosures}

The authors report no conflict of interest concerning the materials or methods used in this study or the findings specified in this paper.

\section{Author Contributions}

Conception and design: both authors. Acquisition of data: both authors. Analysis and interpretation of data: Tamber. Drafting the article: both authors. Critically revising the article: both authors. Reviewed submitted version of manuscript: both authors. Approved the final version of the manuscript on behalf of both authors: Tamber. Statistical analysis: Tamber. Study supervision: Tamber.

\section{Supplemental Information \\ Previous Presentations}

This work was presented as an abstract at the American Association of Neurological Surgeons Annual Meeting, Los Angeles, CA, April 22-26, 2017.

\section{Correspondence}

Mandeep S. Tamber: British Columbia Children's Hospital, Vancouver, BC, Canada. mandeep.tamber@cw.bc.ca. 